\title{
Percutaneous Transpedicular Fixation: Technical tips and Pitfalls of Sextant and Pathfinder Systems
}

\author{
Mohamed M. Mohi Eldin, Ahmed Salah Aldin Hassan \\ Department of Neurosurgery, Faculty of Medicine, Cairo University, Cairo, Egypt
}

Study Design: The efficacy of the operative techniques, possible benefits as well as pitfalls and limitations of the techniques are discussed. Potential drawbacks are also detected.

Purpose: This study aims to report indications, techniques, and our experience with the use of the Sextant and PathFinder percutaneous transpedicular screw fixation systems.

Overview of Literature: Percutaneous pedicle screw insertion is a novel technique. Successful percutaneous placement of pedicle screws requires surgical skill and experience because of lack of anatomic surface landmarks. Fluoroscopy-guided percutaneous placement of pedicle screws is effective. Many systems are now available.

Methods: We conducted a prospective operative and postoperative analysis of 40 patients with absolute indication for thoracic or lumbar instability between January 2009 and June 2013. All procedures were performed with the Sextant (group A) and PathFinder (group B) systems under fluoroscopic guidance. Operative techniques are discussed and the results compared.

Results: Percutaneous transpedicular screw fixation minimizes the morbidity associated with open techniques without compromising the quality of fixation. A total of 190 screws were inserted. There was no additional morbidity. Postoperative computed tomography images and plain X-rays were analyzed. Reduction of visual analog scale scores of back pain was evident.

Conclusions: Fluoroscopy-guided percutaneous pedicular screws are feasible and can be safely done. Current systems allow multisegmental fixation with significantly less difficulties. The described techniques have acceptable intra- and postoperative complication rates, and overall sufficient pain control with early mobilization of patients.

Keywords: Percutaneous; Transpedicular; Screw fixation; Minimally invasive

\section{Introduction}

Percutaneous pedicle screw insertion is novel and recently reported technique. The technique of minimally invasive transpedicular percutaneous screw placement was first performed in the late 1970s and was used only for temporary external fixation of the spine $[1,2]$. The concept was recently changed to percutaneous internal fixation for segmental lumbar instability as a form of minimally invasive spine surgery [3-8]. Successful percutaneous placement of pedicle screws requires surgical skill and experience because of a lack of dorsal anatomic surface landmarks. Fluoroscopy-based guidance is effective and accurate in percutaneous placement of lumbar pedicle screws $[9,10]$. Claimed disadvantages of percutaneous screws include potentially longer operative times,

Received May 31, 2015; Revised Jul 2, 2015; Accepted Jul 3, 2015

Corresponding author: Mohamed M. Mohi Eldin

Department of Neurosurgery, Faculty of Medicine, Cairo University, Cairo, Egypt

Tel: +002-02-33022907, Fax: +002-02-33022907, E-mail: mmohi63@yahoo.com 
the need for a steady learning curve, loss of surgeon control or tactile feel, and an inability to visualize open anatomy. Moreover, the implant can be difficult to compress or distract.

Percutaneous transpedicular fixation systems have become more widely used in recent years mainly for the treatment of dorsal, dorsolumbar, and lumbar instability. Many systems are now available. These include PathFinder (Abbott Spine), Sextant (Medtronic), Apollon (Solco Medical), and CDH Longitude (Medtronic). Only a few studies have been published, most of them focused on technical matters and postoperative assessment of screw placement. Available comparative data about different systems and functional outcome are still sparse. Only three studies have involved the Sextant and PathFinder percutaneous fixation systems for lumbar instability [10-12].

This study aims to report the indications, operative techniques, and our experience with the use of the Sextant and PathFinder percutaneous transpedicular fixation systems in patients with thoracic, thoracolumbar and lumbar instability. The feasibility and efficacy of the presented operative techniques, possible benefits, as well as pitfalls and limitations of the techniques are discussed. Potential drawbacks are considered.

\section{Materials and Methods}

\section{Patients}

\section{1) Patient selection}

The study idea was approved by the Neurosurgery Department Board of Cairo University Hospital, and all patients gave informed consent before being enrolled into the study. The study group was recruited from patients with radiological validated absolute indication for thoracic, thoracolumbar or lumbar instability who had proper diagnostic assessment. Compression fracture, spondylolisthesis, and chronic discogenic pain were the main indications. Conservative therapy had not provided satisfactory pain relief. Patients with diabetes mellitus and patients with more than one pain syndrome were excluded. Exclusion criteria also included inadequate identification of bone anatomy on image intensification, extensive disruption of bone anatomy, prior posterolateral fusion, vertebral rotation-scoliosis, high grade spondylolithesis, severe osteoporosis, and tumor or infection at the instrumented level. Sclerotic hard pedicles were also
Table 1. Indication of surgical fixation

\begin{tabular}{lc} 
Surgical indication & No. of patients \\
Spondylolithesis & 22 \\
Traumatic fracture & 14 \\
\hline Discogenic pain & 4 \\
\hline
\end{tabular}

Table 2. Techniques of pedicle screw insertion

\begin{tabular}{ll} 
Technique of screw insertion & No. of screws \\
Sextant & Group A: 25 cases \\
PathFinder & Group B: 15 cases \\
\hline
\end{tabular}

excluded to avoid difficult or impossible Jamshidi needle placement.

\section{2) Patient population}

We conducted a prospective operative and postoperative analysis of cases with thoracic, thoracolumbar, and lumbar instability. The study included 40 patients (22 females and 18 males) ranging in age from 25 to 70 years (mean age, 44.6 years). The indications for surgical fixation were spondylolithesis $(n=22)$, traumatic fracture $(n=14)$, and chronic discogenic pain $(n=4)$ (Table 1). All cases underwent fluoroscopy-guided percutaneous fixation of the spine between January 2009 and June 2013. All procedures were performed with the Sextant (group A, n=25) and PathFinder (group B, $\mathrm{n}=15$ ) percutaneous fixation systems under fluoroscopic guidance (Table 2).

\section{Equipment and instrumentation}

Equipment needed for percutaneous transpedicular fixation includes a posterior percutaneous fixation system, fluoroscopy unit, Jamshidi (PAK) needles, and a radiolucent operating table. A single C-arm is enough in most of the cases. Bipolar C-arm is used when available. The first version of the Sextant system (Fig. 1) consisted of polyaxial cannulated screws and pre-bended lordotic rods basically designed to accommodate two vertebral levels. In the second iteration, special assembly connectors have been included to accommodate three vertebral levels. The PathFinder system consists of polyaxial cannulated screws, with screw extenders guiding the rod into the screw head utilizing a paramedian approach designed to accommodate three or four vertebral levels. 


\section{Operative technique}

\section{1) Anesthesia}

General intravenous anesthesia (GIVA) is recommended routinely in the thoracic spine, but not necessarily in the lumbar spine. GIVA was used for all but one case. The exception was an elderly female whose general condition contraindicated GIVA. She was sedated with local spinal anesthesia for lumbar fixation.

\section{2) Procedure}

The patient is positioned prone on a radiolucent frame. Attention is paid to ensure that lordosis is maintained, that all bony prominences are padded, and that the abdomen remains freely suspended. The arms are abducted and placed on padded boards. The legs are maintained in extension. A standard dose of Ceftriaxone (Rocephin) $1 \mathrm{~g}$ is administered intravenously before the skin incision.

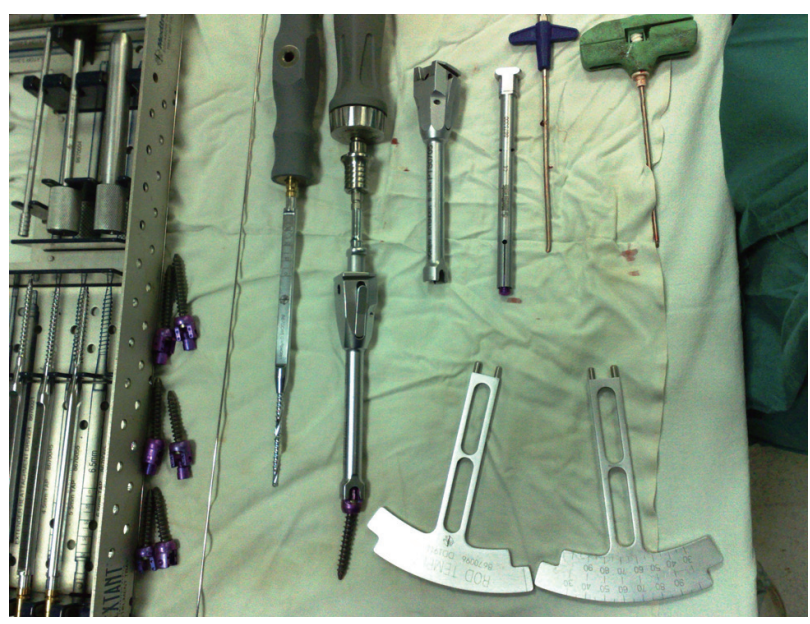

Fig. 1. The Sextant percutaneous stabilization system consists of polyaxial cannulated screws and pre-bended lordotic rods with Jamshidi needles, guide wires, cannulated tap, and special assembly connectors.

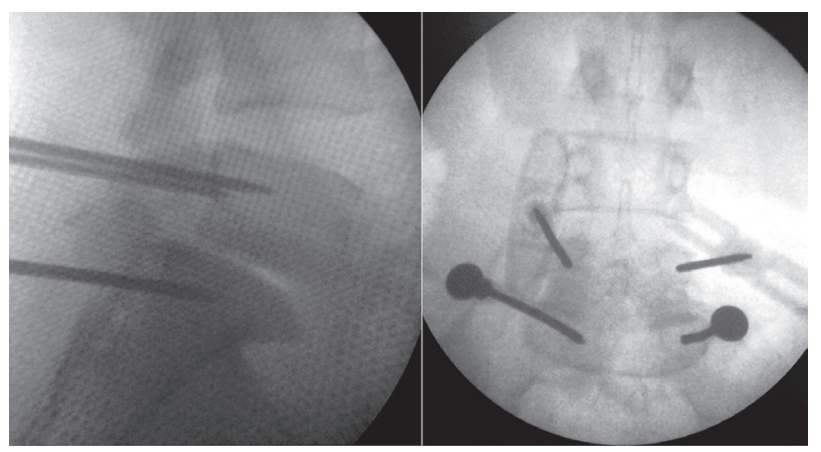

Fig. 2. The Jamshidi needle is advanced into the pedicle with care taken not to advance across the medial border.
Anteroposterior (AP) and lateral fluoroscopic images are obtained, and the vertebrae to be instrumented are determined with the C-Arm and the midline, lateral pedicular, and interpedicular lines marked on the skin. We recommend a more lateral incision lateral to the border of the pedicle on AP imaging for percutaneous transpedicular placement, especially in lumbar vertebrae, to create the right trajectory for placement of instrumentation. The incision should be sufficient to accommodate the screw extender used with the system. A transpedicular channel is created using the Jamshidi needle under AP and lateral fluoroscopic guidance into the upper outer quadrant of the pedicle. The needle is advanced using a mallet into the pedicle approximately $30 \mathrm{~mm}$ into the bone, with care taken not to advance across the medial border (Fig. 2). Guide wires are subsequently inserted into the pedicles through the needles. Insertion is confirmed fluoroscopically. After removal of the needles, atraumatic dilatation of the paraspinal muscles is done using special serial dilators followed by screw extenders over the guide wires. The small and medium dilators are removed, leaving the final dilator (screw extenders) in place. The pedicles and proximal half of the vertebral body are tapped with a cannulated tap. Subsequently, the dilators are removed and the cannulated screws are introduced over the guide wires into their trajectory (Fig. 3). The guide wires are removed,

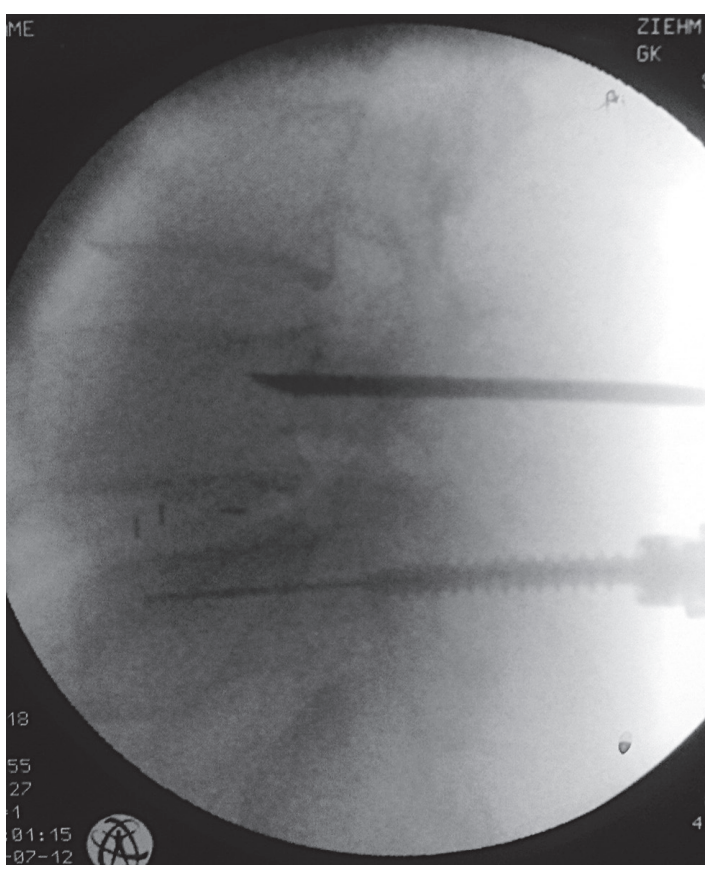

Fig. 3. After removal of the dilators, the cannulated screws are introduced over the guide wires into their trajectory. 
possibly using a Kocher, after the pedicle screw is placed beyond the pedicle. All procedures are done under lateral fluoroscopy. For the Sextant system, the screw extenders are lined up, and a rod measuring device is used to measure the appropriate rod length (Fig. 4). The rod inserter is attached to the screw extenders and the rods are inserted percutaneously using the free-hand technique through a small incision rostral to the proximal screw incision, and passing through the screw head extenders of the proximal and caudal screws (Figs. 5, 6). The screws are then compressed, tightened, and broken off according to the appropriate torque of the locking caps (Fig. 7). For the PathFinder system, bending of the rod prior to implantation is required. The screws are attached to C-shaped

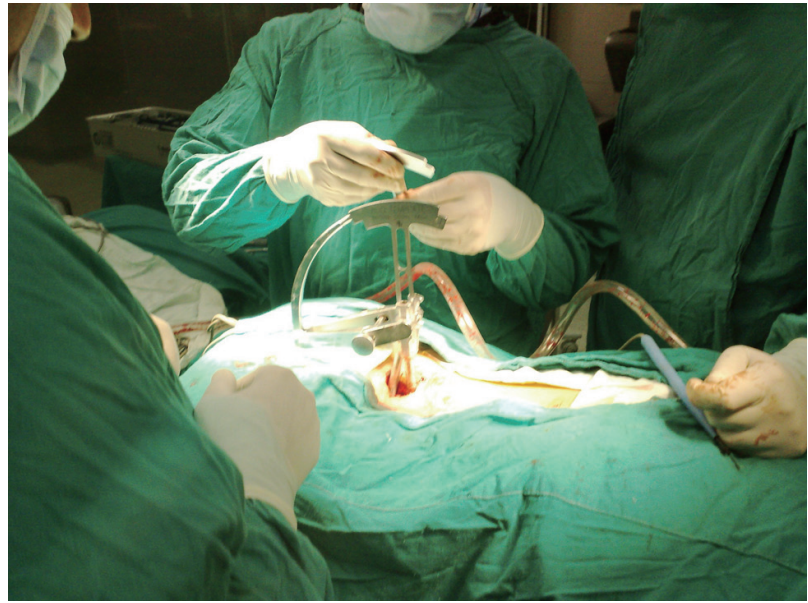

Fig. 4. In the Sextant system, the screw extenders are aligned and a rod measuring device is used to measure the appropriate rod length.

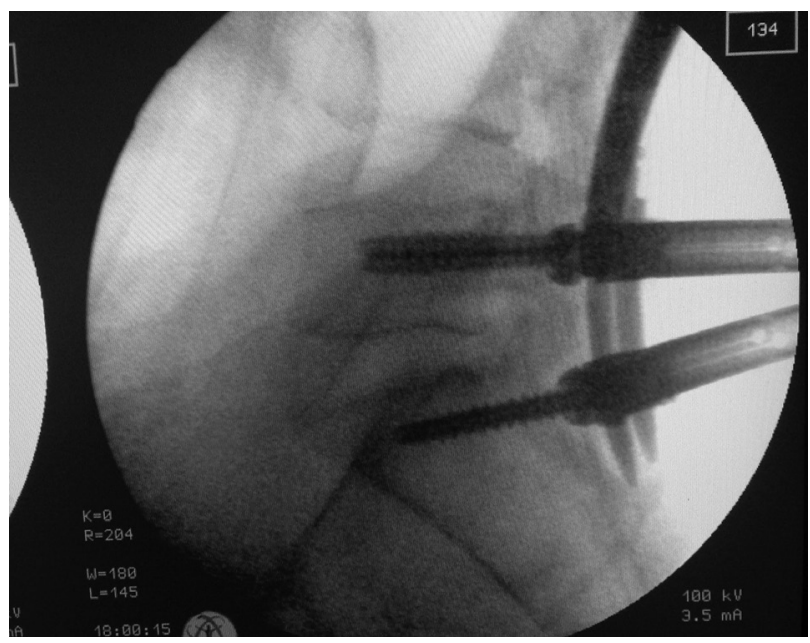

Fig. 5. In the Sextant system, the rod inserter is attached to the screw extenders and the rods are inserted percutaneously, passing through the screw head extenders of the proximal and caudal screws. towers, a slotted extender sleeve that attaches securely to the screw heads and extends above the skin. The rod is then passed down and implanted through connecting the screw snap incisions into one single incision on each side. It is through those towers that the rod is delivered into the screw head deep in the tissue and tightened in place with set screws (Fig. 8). Reduction and compression can

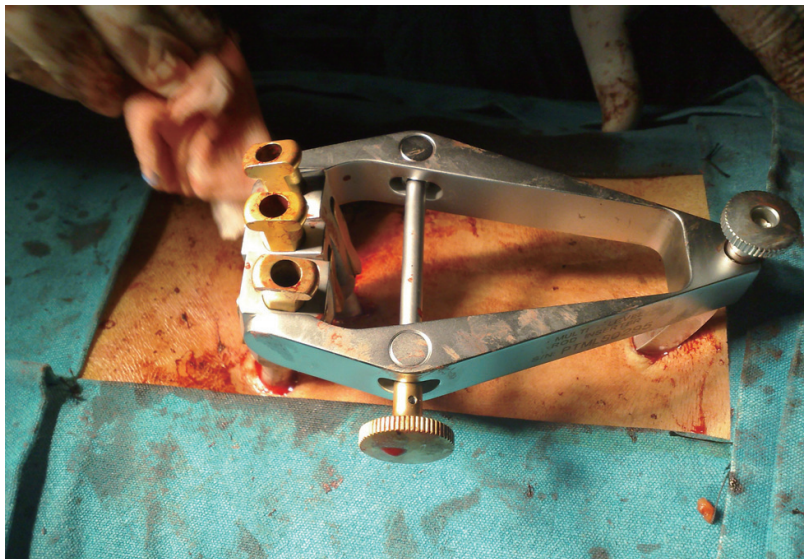

Fig. 6. In the Sextant system, rods are inserted percutaneously using the free-hand technique through a small incision rostral to the proximal screw incision.

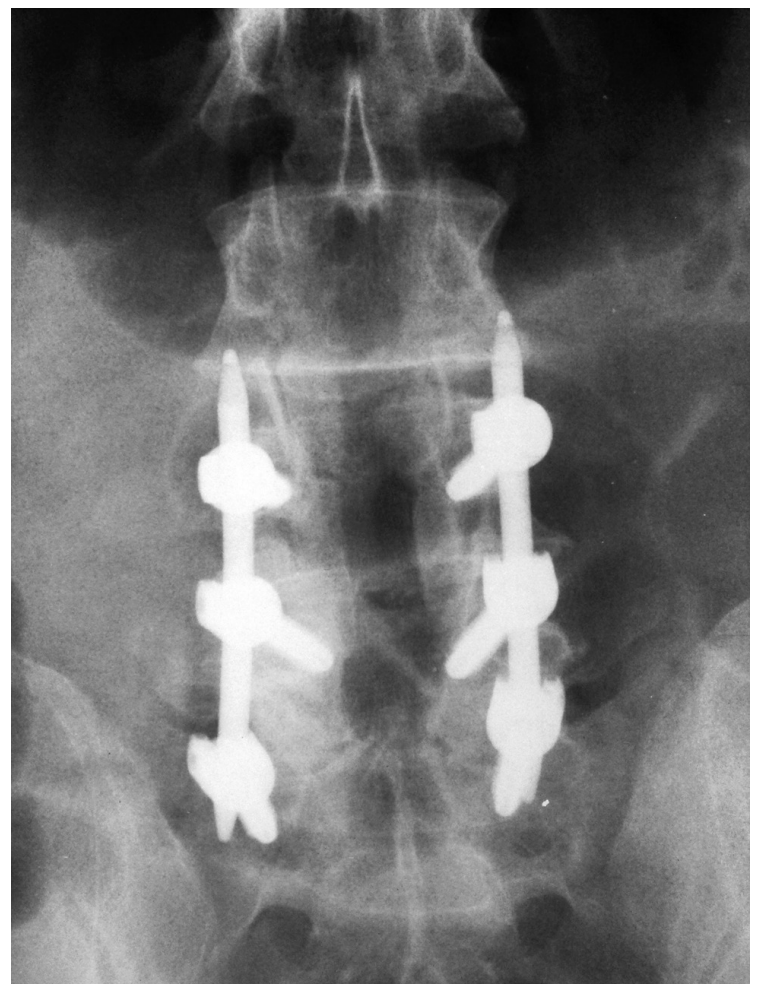

Fig. 7. In the Sextant system, the screws are finally compressed, tightened, and broken off according to the appropriate torque of the locking caps. 
then be performed. Finally, the instruments are removed and skin incisions closed (Fig. 9). When decompression of neural structures is needed, it is limited to the affected levels using the miniopen approach. In this approach, per-

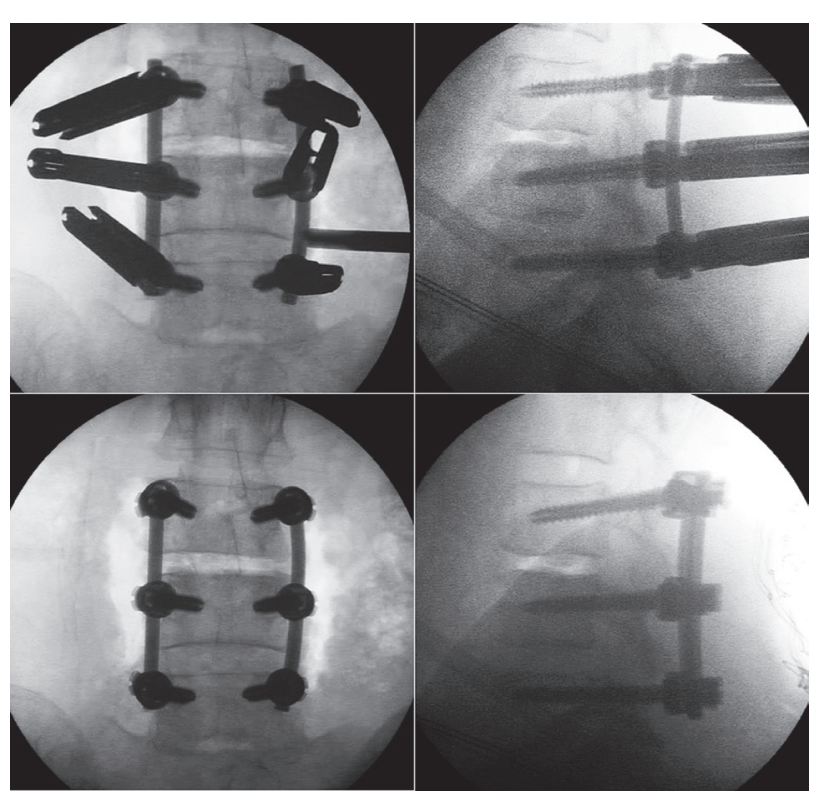

Fig. 8. In the PathFinder system, the rod is delivered through screw towers into the screw head deep in the tissue and tightened in place with set screws.

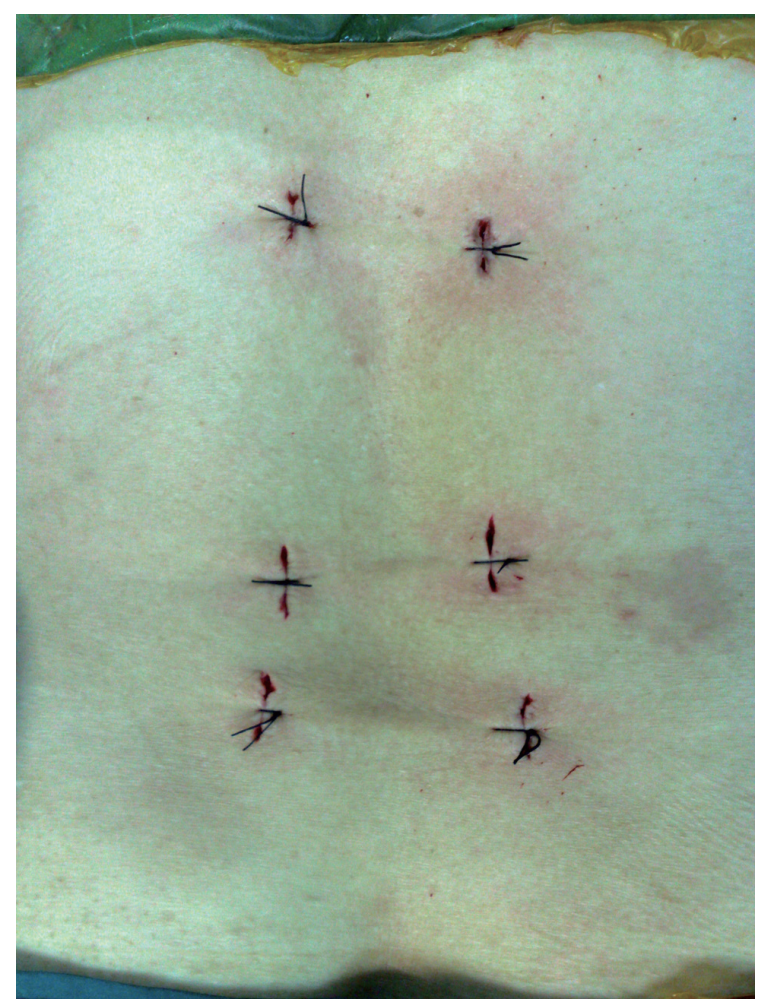

Fig. 9. The instruments are removed and skin incisions closed. cutaneous instrumentation minimizes the skin incision length and muscle dissection (Fig. 10).

\section{3) Postoperative considerations}

Bracing for standalone rods and screws is controversial, especially in trauma cases. To assist in fusion, we usually braced these patients for 3 months after percutaneous transpedicular placement, especially in trauma cases. Early ambulation was encouraged, as it improves prognosis and shortens recovery. Postoperative bracing was always used for instrumentation-mediated fusion. Potential complications of percutaneous screw placement with or without fusion were recorded.

\section{4) Outcome assessment}

Operative techniques, technical tips, and pitfalls were recorded for both systems. Clinical evaluation was done before surgery and before discharge and at 3 months, 6 months, and 1-year. Postoperative routine AP and lateral plain radiographs were performed within 48 hours after surgery. Computed tomography (CT) scan with $2 \mathrm{~mm}$ axial slices with bone window was performed to evaluate implant position after surgery. These images were inspected for evidence of pedicle violation and/or screws malposition. Correlation between clinical symptoms and radiological results were also reported. Follow up ranged between 6 months and 48 months. CT scan with $2 \mathrm{~mm}$ axial slices with bone window was performed in all cases to evaluate implant position after surgery. These images were inspected for evidence of pedicle violation. Correla-

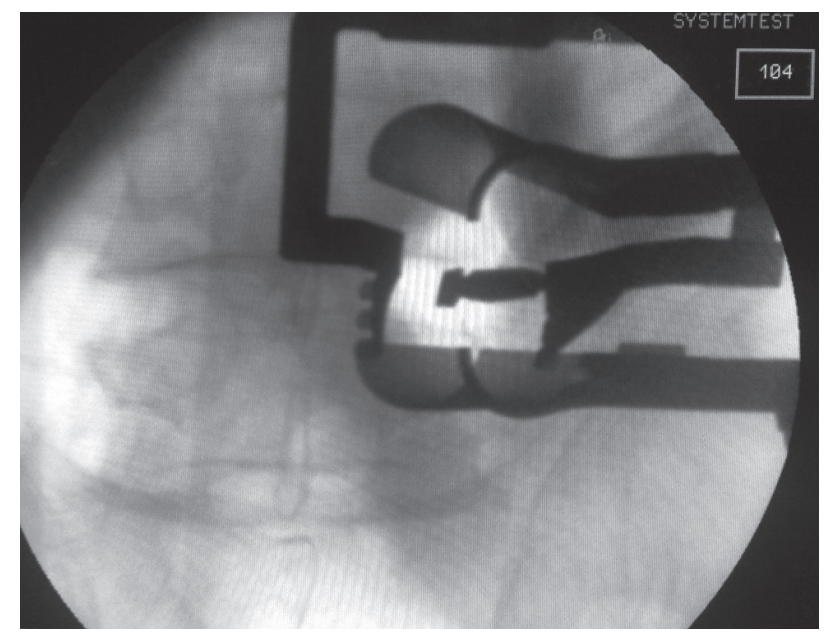

Fig. 10. In the miniopen approach, percutaneous instrumentation minimizes skin incision length and muscle dissection. 
tion between clinical symptoms and radiological violation was assessed.

\section{Results}

\section{Operative results}

All procedures were performed with the Sextant system (group $\mathrm{A}, \mathrm{n}=25$ ) or the PathFinder system (group B, $\mathrm{n}=15$ ) under fluoroscopic guidance. With the PathFinder system, the implantation technique does not differ significantly from that of the Sextant system, except for some technical points. Median surgical time was $115 \pm 42$ minutes with no blood loss. As all percutaneous transpedicular placement steps were fluoroscopy-guided, the quality of fluoroscopy imaging of the spine was anticipated to be very good. Early in the study, we converted one percutaneous procedure to open surgery for fear of violating the insertion guidelines with a bad quality fluoroscopy. This case was excluded from the study. At targeted levels, small incisions directly above the projection of the pedicles on AP imaging were used for dorsal pedicles. However, a more lateral incision was used in lumbar vertebrae, about 1 to $2 \mathrm{~cm}$ lateral to the pedicle projection on AP images, according to the level and the presence or absence of obesity, to be in the right trajectory in line with long axis of the pedicle. In later cases, we cannulated all pedicles on each side, or both pedicles per vertebra simultaneously under AP projection. Lateral projection confirmation was done and guide wires were placed and advanced using the same lateral projection. Strict monitoring of the position was done in an attempt to have full control of the sharp ended guide wire from insertion until removal. Nonetheless, in one patient with osteoporosis, advancement of the guide wire into the abdomen occurred with rapid take out during introduction of the screws. The female patient was closely monitored for signs of blood loss or intestinal injury. No concerns were evident. Sonographic and surgical consultations confirmed the lack of complications. In another case, backward dislodgment occurred while removing the tap. We had to repeat all steps of screw introduction from the beginning. In cases requiring step reduction, as in spondylolithesis, especially in Sextant group, the patient was placed in the best reduction position. This was because the first and second generation of the Sextant system allows only some distraction and compression that may be ineffective in step reduction. However, both the first and second generation PathFinder systems feature a reduction instrument when attached to the screw assembly, which helps in step reduction. With the Sextant system, there were two cases in which the screw extender assembly lost the connection with screw head after removal of the guide wire and during connection of the screw assemblies to the rod trocar. In one case, it was necessary to reapply the guide wire, remove the screw, and redo the steps. In the second case, the guide wire reapplication was difficult and the screw extender assembly lost control over the screw head. The incision was extended, the fascia opened, and dissection performed down to the level of the screw to reach the screw head directly. The Sextant rods are prebent lordotic rods and cannot permit modeling. However, PathFinder rods need modeling before positioning because they do not allow in situ correction. So, bending of the rod was necessary before its implantation, according to the level. During Sextant rod insertion, a palpable engagement was confirmed with the rod tester. In two cases, properly placed rods on lateral view images proved to be out of one of the screw heads on AP view images (Fig. 11). In the two cases, rod reintroduction was done and verified by both AP and lateral fluoroscopic images before tightening the caps of the screw heads.

\section{Clinical results}

Postoperative recovery during the up to 2year follow-up was rapid for all patients. Clinical results were satisfactory in all cases. There was a significant decrease of back pain quantified by patient self-assessment using a standard visual analog scale (VAS) score. In all patients, pain was recorded as absent or very mild (VAS 89). Images taken at the 1-year follow-up showed no signs of screw loosening

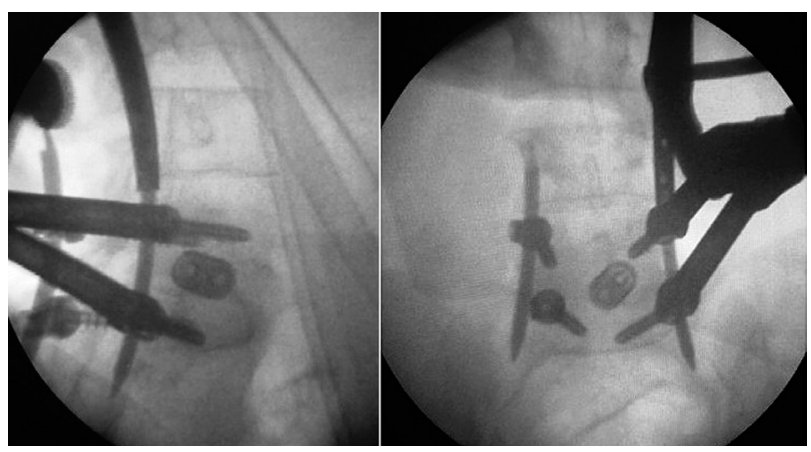

Fig. 11. During Sextant rod insertion, properly placed rods on lateral view images may prove to be out of one of the screw heads on anteroposterior view images. 
Table 3. Total number of screws

\begin{tabular}{ccc} 
Total no. of screws & Screws completely within pedicle & Screws with cortical breaching \\
\hline 190 & 149 & 41 \\
\hline
\end{tabular}

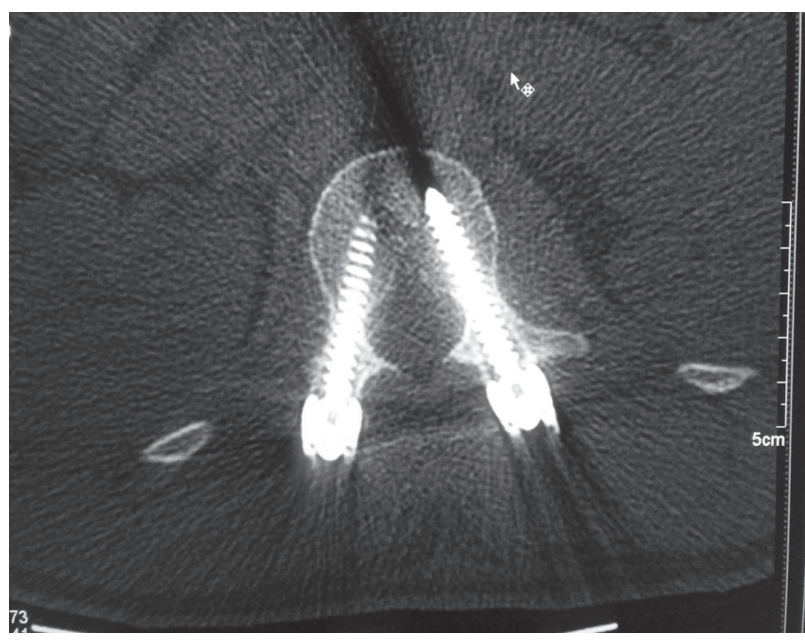

Fig. 12. Out of 190 screws, 149 were placed completely within the pedicle unit.

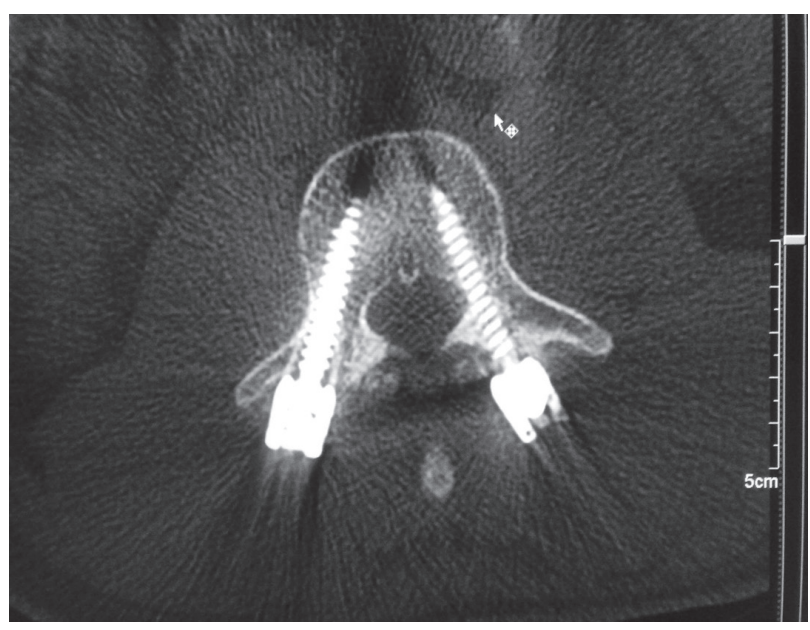

Fig. 13. Out of 190 screws, 36 were displaced less than $2 \mathrm{~mm}$ (minor displacement).

(radiolucency around screws) or signs of implant fracture or failure. Patients were mobilized in the first postoperative week. A rigid brace was used in all cases for 6 weeks. In the reported follow-up none of the patients required revision surgery as a result of instrumentation failure.

\section{Radiological results}

The accuracy of pedicle screws was assessed using thin slice CT scan bone window to evaluate even minimal

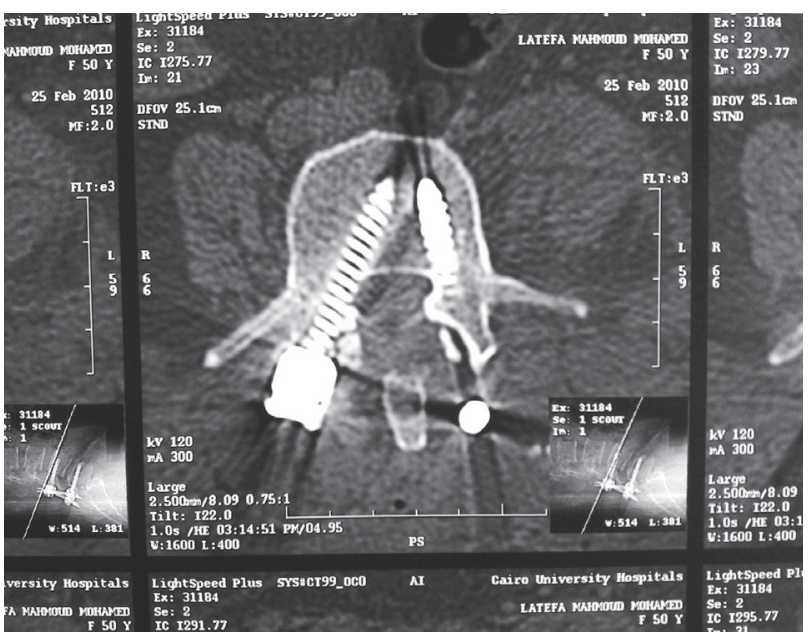

Fig. 14. Out of 190 screws, five were displaced between $2 \mathrm{~mm}$ and 4 $\mathrm{mm}$ (moderate displacement).

Table 4. Degree of screw displacement

\begin{tabular}{lcc} 
Degree & $\begin{array}{c}\text { No. of screws } \\
(\%)\end{array}$ & $\begin{array}{c}\text { Displacement in } \mathrm{mm} \\
\text { Minimal }\end{array}$ \\
Moderate & $56(18)$ & $<2 \mathrm{~mm}$ \\
Sever & 0 & $2-4 \mathrm{~mm}$ \\
\hline
\end{tabular}

Table 5. Direction of cortical perforation

\begin{tabular}{lc} 
Direction of perforation & No. of screws $(\%)$ \\
Superior & 0 \\
\hline Inferior & 0 \\
Medial & $21(11.1)$ \\
\hline Lateral & $20(10.5)$ \\
\hline
\end{tabular}

cortical breaching. A total of 190 screws were inserted; 149 screws were placed completely within the pedicle unit (Table 3, Fig. 12). Cortical breaching of pedicles occurred with $41(21.6 \%)$ screws. Screw displacement assessed by CT scan was minor $(<2 \mathrm{~mm})$ for 36 screws (Fig. 13) and moderate (2-4 mm) for five screws (Fig. 14). Displacement exceeding $4 \mathrm{~mm}$ was not reported (Table 4 ). CT assessment of the direction of screw displacement was also assessed. Twenty screws were laterally deviated and 21 displayed medial perforation (Table 5). 


\section{Complications}

None of the patients developed additional neurological deficits related to the instrumentation. The postoperative clinical development of new symptoms related to displaced screws were reported in only one case that required open correction on postoperative day 2. There were no cases of newly developed neurological injury. Fracturing of the pedicle during screw insertion was not reported. No wound infections occurred. No further complications were detected during the follow-up period. No surgeryrelated neurological complications, implant-related complications, dural tear, or wound infections were observed. No monoaxial screws were used. Polyaxial screws were used by default, despite the proposed slight movement, also after implantation, between the head and the arm of the screw, with stability of the operated segments as confirmed by follow-up dynamic X-rays. There were three female patients with osteoporosis. In those cases we used fenestrated screws with cement augmentation before rod placement (Fig. 15). No postoperative loss of correction or stabilization was reported. Implant prominence in thin individuals were not reported, and no cases needed reoperation for this reason.

\section{Discussion}

Minimally invasive spine surgery is becoming the treatment of choice for many spinal conditions including

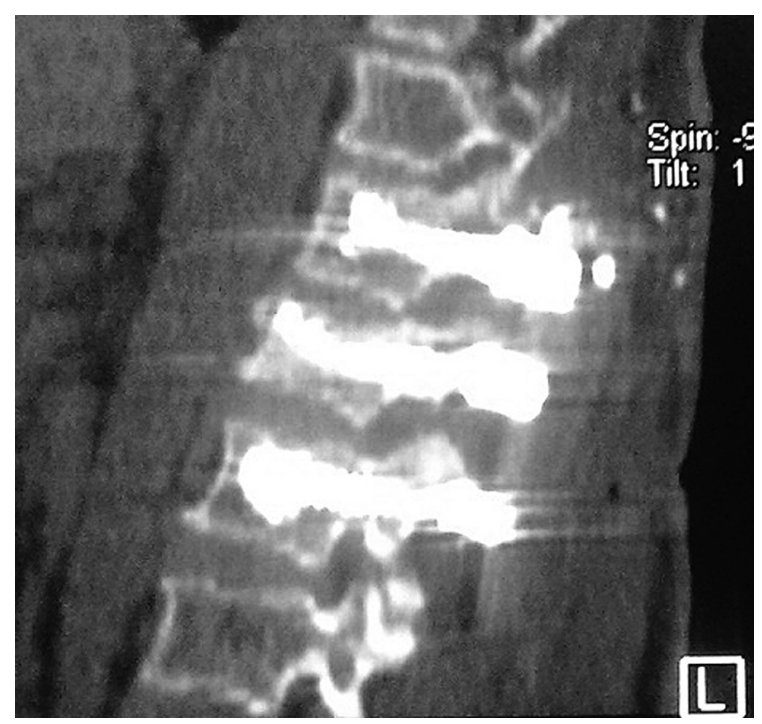

Fig. 15. Fenestrated screws with cement augmentation before rod placement in patients with osteoporosis. degenerative and traumatic disorders. Recent developments have allowed for the preservation of normal spinal anatomical structures when performing spinal fusion and instrumentation. These advances help to preserve muscles, ligaments, and bony structures of the spine that are critical to spinal function and improved outcomes. Additionally, neural innervations and vascular supply to the paraspinal muscles and facet joints are preserved, potentially reducing adjacent segment disease in patients undergoing spinal fusion and instrumentation. The goal is to develop treatment methods that are less painful, more cost effective, and longer lasting, and which improve the quality of life and allow a rapid return to daily activities $[4,6,7,13,14]$.

From our point of view, conventional procedures carry considerable risks as reported in the literature. Interest in minimally invasive posterior internal fixation and fusion has recently increased slowly until technological advances facilitated safe and effective procedures. Percutaneous implantation of transpedicular screws based exclusively on image guidance constitutes a clinical and surgical challenge. Many percutaneous fixation systems have been introduced in spine practice. Unlike more traditional pedicle screw systems, the bony anatomy of the spine does not need to be exposed. This facilitates more rapid returning to work or activities of daily living [14-19].

Here, we present our data with the fluoroscopic guided implantation of two widely-used percutaneous systems. When decompression of neural structures was needed, it was limited to the affected levels, with the miniopen approach. In this approach, percutaneous instrumentation minimized the skin incision length and muscle dissection.

Several recent approaches to minimally invasive posterior fusion with internal fixation have been described. In 2000, Muller et al. [20] described a "keyhole" approach for endoscopically assisted pedicle screws fixation. The screws were connected by rods bluntly tunneled between the screws. The ports needed to be removed in a blind procedure to pass the rod between the screws. Foley and Gupta [7], and Foley et al. [8] described an internal fixation technique called the Sextant procedure by its manufacturer, Medtronic Sofamor Danek. This equipment is used to percutaneously insert pedicle screws and accurately connect them with rods. The primary application is internal fixation to supplement an anterior lumbar interbody fusion or posterior lumbar interbody fusion; there is no provision for posterolateral fusion. Like the Muller 
technique, the Sextant procedure involves blind tunneling for the rod passage with no provision for hemostasis in the muscle. Six small incisions are needed for a one-level fusion. Internal fixation for a two-level fusion can be provided by placing screws in the end vertebrae, skipping the intervening vertebra.

Spinal Concepts introduced the Pathfinder system for minimally invasive posterior internal fixation [21]. The system is similar to the Sextant approach in that it allows accurate blind placement of the rod through the pedicle screws. Rather than the multiple small incisions for Sextant patients, Pathfinder-treated patients receive one larger incision over the pedicles on each side. The surgical field is limited with the Pathfinder technique, similar to working through the early Kambin straight cannula. Both the Sextant and the Pathfinder techniques depend heavily on intraoperative fluoroscopy.

The PathFinder system using fluoroscopic guidance has a unique design, which is simple, easy-to-use, and extremely versatile. The system uses a modified Wiltse technique that combines the familiarity of an open procedure with the patient benefits of a minimally invasive approach. The PathFinder system represents the first minimally invasive surgical system capable of spinal reduction, graft compression, segmental distraction, and multi-segmental instrumentation through two small paraspinal skin incisions. Maintenance of normal anatomical integrity will ideally lead to better success rates as well as a reduced incidence of adjacent segment disease, and hence improved patient outcomes.

PathFinder's features include traditional top-down rod placement through a familiar muscle-splitting and sparing Wiltse approach. Additional features include new implants and instruments, including $4.5 \mathrm{~mm}$ screws, awl taps for one-step tapping, improved reduction knobs for a controlled reduction up to $20 \mathrm{~mm}$ and a unique revision tool to simplify the removal process if necessary. The features are part of a complete minimally invasive surgical portfolio, including Harmony Posterior instruments, Harmony Retractor and Harmony Port Systems, as well as a variety of interbody options.

\section{Technical tips and pitfalls}

The operative technique of the PathFinder and Sextant systems is similar. The percutaneous implantation technique is common to both systems. However, some of the technical details need to be discussed.

All placement steps are fluoroscopy-guided. Any concern for the quality of fluoroscopy imaging of the spine should prompt the decision to perform open surgery. To save time and reduce radiation exposure, we prefer to cannulate all pedicles, at least on each side, using AP projection with confirmation provided using lateral projection. Guide wires are then placed and advanced using the same lateral projection.

The patient needs to be positioned in the best reduction position for the treated pathology, because the percutaneous systems allow only some distraction and compression that may be ineffective in step reduction.

It is vital to monitor the position and have full control of the guide wire from insertion to removal, with special care taken to avoid advancement of the guide wire while introducing the tap and screws, or backward dislodgment while removing the tap. Inadvertent guide wire advancement anteriorly increases the risk of intra-abdominal injury to vessels, gut, and other structures. Easy loose wire movement and advancement may warrant trajectory revision, as it carries the possibility of being outside the vertebral body.

Fluoroscopic guidance of screw insertion at the targeted levels through the small incisions is useful. Some surgeons use an incision above the projection of the pedicles on AP imaging for percutaneous transpedicular screw placement. This is particularly good in dorsal pedicles. However, we recommend a more lateral incision in lumbar vertebrae, which will create the right trajectory for instrumentation placement in line with long axis of the pedicle $[11,12,21,22]$. Although two adjacent pedicles can be accessed through a single larger incision, we do not recommend larger incisions. A single midline incision has been advocated by other authors, with the fascia being kept intact and opened only for screw placement, but we did not utilize that approach. Care should be taken not to dislodge the guide wire while removing the Jamshidi needles, taps, and other materials.

The PathFinder fixation system has smaller diameter pedicular screws available for use in the thoracic pedicles. The Sextant system does not have this option, rendering its use in the thoracic spine limited.

During Sextant rod insertion, although a palpable engagement is confirmed with the rod tester, the optimal placement of the rods through the screw heads should be verified by both AP and lateral fluoroscopic images before 
tightening. A single AP or lateral view may be misleading (Fig. 11).

In the Sextant system, if there is a problem with a screw extender or screw head, the guide wire can be reapplied, the screw removed, and the steps repeated, if possible. If there is still a difficulty in seating the rod, consideration should be made for extending the procedure to an open one. The chance to convert the minimally invasive style into an open conventional style at a given trajectory is always possible by extending the skin incision and opening up the fascia and dissection down to the level of the rod and screw. In this manner, open intervention under direct vision can be performed with direct open seating of a rod into the screw head. Open surgery always remains a solution if there is a problem.

The PathFinder fixation system needs modeling of the rod before its positioning because it does not allow in situ correction. Bending of the rod before its implantation according to the level is required. The Sextant rods are prebended lordotic rods and cannot permit modeling.

In our judgment, use of the Sextant system in the thoracic spine is justified mostly in patients in whom open surgery carries unacceptable risks. Patients who need short term segmental stability, as in the infectious spondylodiscitis or metastatic disease, are good candidates for the procedure.

There are several limitations and drawbacks concerning the use of the Sextant in the thoracic region. Since the system was developed for minimally invasive procedures of the lumbar spine, only pre-bent lordotic rods are currently available. Implantation of lordotic rods with the Sextant arm in patients with marked kyphosis can be technically demanding or even theoretically impossible. Thorough evaluation of preoperative CT and magnetic resonance images with regard to the degree of kyphosis at the affected levels is mandatory to avoid intraoperative problems with the system. Percutaneous implantation of lordotic rods may also be troublesome in very thin patients. Therefore, in patients with significant kyphotic deformity and/ or cachexia, percutaneous systems with straight rods offer a theoretical advantage and should be considered.

Percutaneous pedicle instrumentation with the use of the Sextant system is feasible in thoracic spine although restricted to select cases. This is due to the lordotic curve of the Sextant rods manufactured to both match the curvature of the lumbar spine and enable in situ assembling rods and screws.

\section{Theoretical tips}

Theoretically, percutaneous pedicle screw placement should markedly minimize inaccurate screw placement. Otherwise, there is no benefit of the procedure. Inaccurate screw placement can result in serious neurologic or vascular, viscus, or pulmonary injury.

Should posterior fusion need to be performed, the simplest type of posterior fusion is facet fusion. This can be performed by connecting the incisions about the adjacent screw heads on each side and exposing the facet. The facet joint cavity is decorticated and impacted in compression with local bone autograft, allograft, or artificial bone chips and granules, which can be used to obtain a facet fusion. The same route could be utilized to do minimally invasive posterior lumbar interbody fusion or even transforaminal lumbar interbody fusion (Fig. 3).

Rapid postoperative recovery, less postoperative pain, and better function means that the theoretical benefits of have been achieved. These result in less damage, less atrophy, and less edema to the back muscles. The back muscle (multifidus) contributes more than two-thirds of the stiffness (resistance to flexion/extension, lateral bending, and rotation) of the lumbar spine [15].

\section{Potential complications}

Regarding the need for fusion, biomechanically the survival of the implant is questionable. In our series of posterior percutaneous fixation without fusion, we did not recognize any breakages, especially in cases of vertebral fractures. No painful implant-related complications reported. The need to remove the implant once the fracture heals is a matter of debate.

Potential complications of percutaneous screw placement with or without fusion include instrumentation misplacement, infection, bleeding, pseudoarthrosis, and adjacent segment degeneration. Complications of minimally invasive posterior fixation reported in the literature included screw misplacement requiring revision, neurological injury, radicular pain, instrumentation prominence, and wound infection $[7,10,13,15,19]$. In our series, the postoperative clinical development of new symptoms related to displaced screws were reported in only one case that required open correction on postoperative day 2 .

Despite the validated efficacy of intraoperative fluoroscopic guidance in spinal procedures, concerns are 
still evident and include the prolonged surgical time, increased exposure to radiation, and surgeon's learning curve $[5,11,13]$.

\section{Conclusions}

The percutaneous approach provides good primary stabilization of the spine, prevents further loss of the correction, and ensures a rapid recovery with good clinical outcome. Current systems allow multi-segmental fixation with significantly less difficulties. The described techniques have an acceptable intra- and postoperative complication rate and overall sufficient pain control with early mobilization of patients.

\section{Conflict of Interest}

No potential conflict of interest relevant to this article was reported.

\section{References}

1. Magerl F. Injuries of the thoracic and lumbar spine. Langenbecks Arch Chir 1980;352:428-33.

2. Magerl FP. Stabilization of the lower thoracic and lumbar spine with external skeletal fixation. Clin Orthop Relat Res 1984;(189):125-41.

3. Kambin P. Posterolateral percutaneous lumbar interbody fusion. In: Kambin P, editor. Arthroscopic microdiscectomy: minimal intervention in spinal surgery. Baltimore: Urban \& Schwarzenburg; 1991. p.117-21.

4. Leu H, Hauser R, Schreiber A. Percutaneous lumbar spine fusion. Acta Orthop Scand Suppl 1993;251:1169.

5. Vaccaro AR, Rizzolo SJ, Balderston RA, et al. Placement of pedicle screws in the thoracic spine. Part II: an anatomical and radiographic assessment. J Bone Joint Surg Am 1995;77:1200-6.

6. Lowery GL, Kulkarni SS. Posterior percutaneous spine instrumentation. Eur Spine J 2000;9 Suppl 1:S126-30.

7. Foley KT, Gupta SK. Percutaneous pedicle screw fixation of the lumbar spine: preliminary clinical results. J Neurosurg 2002;97(1 Suppl):7-12.

8. Foley KT, Holly LT, Schwender JD. Minimally invasive lumbar fusion. Spine (Phila Pa 1976) 2003;28(15
Suppl):S26-35.

9. Acosta FL Jr, Thompson TL, Campbell S, Weinstein $\mathrm{PR}$, Ames CP. Use of intraoperative isocentric C-arm 3D fluoroscopy for sextant percutaneous pedicle screw placement: case report and review of the literature. Spine J 2005;5:339-43.

10. Holly LT, Foley KT. Three-dimensional fluoroscopyguided percutaneous thoracolumbar pedicle screw placement: technical note. J Neurosurg 2003;99(3 Suppl):324-9.

11. Khoo LT, Palmer S, Laich DT, Fessler RG. Minimally invasive percutaneous posterior lumbar interbody fusion. Neurosurgery 2002;51(5 Suppl):S166-81.

12. Maciejczak A, Barnas P, Dudziak P, Jagiello-Bajer B, Litwora B, Sumara M. Posterior keyhole corpectomy with percutaneous pedicle screw stabilization in the surgical management of lumbar burst fractures. Neurosurgery 2007;60(4 Suppl 2):232-41.

13. Ringel F, Stoffel M, Stuer C, Meyer B. Minimally invasive transmuscular pedicle screw fixation of the thoracic and lumbar spine. Neurosurgery 2006;59(4 Suppl 2):ONS361-6.

14. Sasso RC, Best NM, Potts EA. Percutaneous computer-assisted translaminar facet screw: an initial human cadaveric study. Spine J 2005;5:515-9.

15. Weber BR, Grob D, Dvorak J, Muntener M. Posterior surgical approach to the lumbar spine and its effect on the multifidus muscle. Spine (Phila Pa 1976) 1997; 22:1765-72.

16. Spoonamore M. Pedicle-targeting techniques for minimally invasive lumbar fusions. In: Lewandrowski K, Yeung CA, Spoonamore MJ, McLain RF, editors. Minimally invasive spinal fusion techniques. Armonk: Summit Communications; 2008. p.37-47.

17. Wiesner L, Kothe R, Schulitz KP, Ruther W. Clinical evaluation and computed tomography scan analysis of screw tracts after percutaneous insertion of pedicle screws in the lumbar spine. Spine (Phila Pa 1976) 2000;25:615-21.

18. Lonner BS. Emerging minimally invasive technologies for the management of scoliosis. Orthop Clin North Am 2007;38:431-40.

19. Wild MH, Glees M, Plieschnegger C, Wenda K. Fiveyear follow-up examination after purely minimally invasive posterior stabilization of thoracolumbar fractures: a comparison of minimally invasive percutaneously and conventionally open treated patients. 
Arch Orthop Trauma Surg 2007;127:335-43.

20. Muller A, Gall C, Marz U, Reulen HJ. A keyhole approach for endoscopically assisted pedicle screw fixation in lumbar spine instability. Neurosurgery 2000;47:85-95.

21. Khoo LT. Rationale for minimally invasive spine surgery. In: Perez-Cruet MJ, Khoo LT, Fessler RG, editors. An anatomic approach to minimally invasive spine surgery. St. Louis, Mo.: Quality Medical Pub.; 2006. p.89-102.

22. Scheufler KM, Dohmen H, Vougioukas VI. Percutaneous transforaminal lumbar interbody fusion for the treatment of degenerative lumbar instability. Neurosurgery 2007;60:203-12. 